\title{
Structural Changes in Thin Keratoconic Corneas Following Crosslinking with Hypotonic Riboflavin: Findings on In Vivo Confocal Microscopy
}

\author{
Aalia Rasool Sufi ${ }^{1}$, MD; M. Soundaram ${ }^{1}$, MD; Nilam Gohil ${ }^{1}$ MD; Jeremy D. Keenan ${ }^{2,3}$, MD \\ N. Venkatesh Prajna', MD

\begin{abstract}
${ }^{1}$ Department of Cornea and Refractive Surgery, Aravind Eye Hospital, Madurai, India
${ }^{2}$ Francis I. Proctor Foundation, University of California, San Francisco
\end{abstract} \\ ${ }^{3}$ Department of Ophthalmology, University of California, San Francisco, California, USA
}

ORCID:

M. Soundaram: https://orcid.org/0000-0002-5823-4470

\section{Abstract}

Purpose: To report structural changes observable in in vivo confocal microscopy (IVCM) in keratoconic corneas $<400 \mu \mathrm{m}$ treated with hypotonic riboflavin and collagen crosslinking (CXL).

Methods: Ten eyes of ten patients with progressive keratoconus and corneal thickness between 350 and $399 \mu \mathrm{m}$ underwent CXL with hypotonic riboflavin. IVCM was performed preoperatively and at one month, three months, and six months after the procedure.

Results: IVCM analysis one month postoperatively showed complete absence of the subepithelial nerve plexus with gradual regeneration over six months in 8 of the 10 eyes, and poor regeneration in the remaining 2 eyes. The anterior stroma showed extracellular lacunae and hyper-reflective cytoplasm in a honeycomb appearance signifying edema at one month which gradually decreased over six months post $\mathrm{CXL}$. Stromal keratocyte apoptosis was evident in the anterior stroma in all cases and extended to the posterior stroma in four eyes with gradual regeneration evident at three and six months. The specular endothelial count decreased by $8 \%(P=0.005)$ post-CXL, but no corneas developed clinical signs of endothelial trauma.

Conclusion: IVCM analysis of thin corneas after hypotonic CXL showed posterior corneal structural changes. Posterior stromal changes were accompanied by a decrease in the endothelial cell count. This case series was a preliminary feasibility study that might necessitate conducting a well-designed controlled study.

Keywords: Collagen Crosslinking; Hypotonic Riboflavin; In Vivo Confocal Microscopy; Keratoconus

J Ophthalmic Vis Res 2021; 16 (3): 325-337 


\section{INTRODUCTION}

Keratoconus is a noninflammatory degenerative condition of the cornea, characterized by progressive corneal thinning and steepening. ${ }^{[1]}$ Though the exact etiology is unknown, alterations in the corneal collagen structure ${ }^{[2,3]}$ and degree of collagen crosslinking (CXL) leads to a structurally weakened corneal tissue. ${ }^{[4]}$ The spectrum of disease can range from mild astigmatism to severe ectasia requiring corneal transplantation.

CXL is a popular method for management of keratoconus. It is a simple technique in which riboflavin and ultraviolet $A$ (UVA) light is applied to the eye; this treatment is postulated to increase the biochemical and biomechanical stability of the cornea ${ }^{[5]}$ by stimulating the CXL. ${ }^{[6,7]}$ Numerous studies have shown that CXL is a safe method that delays or halts the progression of corneal ectasia and reduces the demand for keratoplasty. ${ }^{[8-10]}$ The major limitation of CXL has been the potential for endothelial trauma when used in thin corneas, usually defined as those $<400 \mu \mathrm{m} .^{[11]}$ To address this shortcoming, different modifications of the CXL procedure for thin corneas have been studied, including contact lens-assisted CXL (CACXL), CXL with customized epithelial debridement, and the use of hypotonic riboflavin. Hypotonic riboflavin hydrates the stromal corneal layer, which should theoretically prevent penetration of the $C X L$ treatment to the endothelium. ${ }^{[12]}$

The morphological effects of CXL on the cornea can be evaluated by in vivo confocal microscopy (IVCM), which is a noninvasive method used to study the living cornea at the cellular level. ${ }^{[13,14]}$ Several studies have demonstrated significant tissue alterations on confocal microscopy following standard CXL for keratoconus, including more compact lamellar interconnections and biosynthesis of new collagen. ${ }^{[15-17]}$ These changes have been theorized to cause strengthening of the corneal collagen. ${ }^{[18]}$ Although the results of IVCM

Correspondence to:

M. Soundaram, MD. Aravind Eye Hospital, Anna Nagar, Madurai 625020, Tamil Nadu, India.

E-mail: drsoundaram123@gmail.com

Received: 28-10-2020Ａccepted: 29-04-2021

Access this article online

Website: https://knepublishing.com/index.php/JOVR

DOI: 10.18502/jovr.v16i3.9429 study of the structural modifications in corneas thinner than 400 microns following $\mathrm{CACX}^{[19]}$ and the customized epithelial debridement ${ }^{[20,21]}$ have already been reported, the IVCM findings following hypotonic CXL have not yet been described. The aim of our study was to analyze the corneal structural modifications observable on IVCM following treatment with hypotonic riboflavin and subsequent CXL.

\section{METHODS}

This prospective study was conducted at Aravind Eye Hospital Madurai, India and adhered to the tenets of Helsinki. Ethical approval was obtained from the institutional review board of Aravind Eye Hospital Madurai. A written informed consent was obtained from all patients before inclusion in the study. Patients who were at least 10 years of age and had keratoconus with preoperative thinnest pachymetry between 350 and $399 \mu \mathrm{m}$ (assessed with Pentacam, Oculus Optikgeräte $\mathrm{GmbH}$, Wetzlar, Germany) and automated endothelial cell density of 2400 cells $/ \mathrm{mm}^{2}$ or higher (assessed with Konan Specular Microscope X NSP-9900, Konan Medical, Inc., Japan) were eligible for inclusion in the study. Corneas were excluded from the study if any of the following factors were present: corneal scar, history of previous anterior segment surgery, active anterior segment disease possibly affecting the epithelial healing, or active allergic ocular disease. Patients who were pregnant, lactating, or had systemic connective tissue disease were also excluded. Only one eye per patient was included in the study; the selection of which eye to include was based on ocular exclusion criteria, or if both eyes were eligible, then the eye with thinner corneal pachymetry was selected.

\section{Ocular Assessment}

All patients were subjected to a comprehensive preoperative ocular examination, which included

This is an open access journal, and articles are distributed under the terms of the Creative Commons Attribution-NonCommercial-ShareAlike 4.0 License, which allows others to remix, tweak, and build upon the work non-commercially, as long as appropriate credit is given and the new creations are licensed under the identical terms.

How to cite this article: Sufi AR, Soundaram M, Gohil N, Keenan JD, Prajna NV. Structural Changes in Thin Keratoconic Corneas Following Crosslinking with Hypotonic Riboflavin: Findings on In Vivo Confocal Microscopy. J Ophthalmic Vis Res 2021;16:325-337. 
measurement of uncorrected distance visual acuity (UCVA), best corrected distance visual acuity (BCVA) and the manifest refraction, as well as an anterior segment slit lamp evaluation and intraocular pressure measurement by noncontact tonometry. Topography and optical pachymetry were then performed using the Pentacam, followed by IVCM using the Heidelberg Retinal Tomograph III equipped with Rostock Corneal Module (HRTIII-RCM; Heidelberg Engineering $\mathrm{GmbH}$, Heidelberg, Germany) and automated endothelial cell density estimation with the Konan Specular Microscope. After completing all tests, a dilated fundus evaluation was performed to rule out any posterior segment pathology. All examinations were repeated in the same fashion at one, three, and six months postoperatively.

\section{Confocal Microscopy}

IVCM was performed with the laser scanning HRT III-RCM, an instrument whose transverse and longitudinal resolution is $2 \mu \mathrm{m}$ and $4 \mu \mathrm{m}$, respectively. Local anesthetic $(0.5 \%$ proparacaine solution, Aurolab, Madurai) was instilled in the eye and patients were asked to look at an external fixation target. The instrument objective was brought into contact with the center of the cornea using a disposable sterile polymethylmethacrylate (PMMA) cap filled with a highviscosity coupling agent (Genteal gel; Novartis pharmaceuticals, Australia). Images of the entire anteroposterior extent of the cornea were taken at the optical center of the cornea. Each individual scan provided a cube of 40 stacked $400 \times 400$ $\mu \mathrm{m}$ scans measuring approximately 80 microns in depth. Two anterior to posterior scans per patient were taken and the best sequence was selected. An area of inferior peripheral cornea outside the treatment zone was scanned in the same manner to serve as a control.

\section{Qualitative Confocal Image Analysis}

Two independent examiners with clinical experience in treating keratoconus and interpreting in vivo confocal images (ARS, MS) reviewed all scans from each study subject, masked to the results of the other examiner and to whether or not the eye had vernal keratoconjunctivitis (VKC) at all visits. Each examiner provided a qualitative assessment of the epithelium, sub-basal nerve plexus, stroma, and endothelium. The graders met before starting their analyses to reach common definitions for epithelial metaplasia, microdot globular cells, dendritic cells, hyperreflectivity, keratocytes, and hyperreflective bands, based on previous publications. ${ }^{[15-17,22,23]}$ The first three clear images immediately posterior to Bowman's membrane were defined as the anterior corneal stromal images, and the first three clear images immediately anterior to the endothelium were defined as the posterior corneal stromal images for analysis. Blurred or non-tangential images were excluded.

\section{Quantitative Confocal Image Analysis}

All selected images were de-identified and randomized. Quantitative analysis was subsequently performed by the same two aforementioned graders using the manufacturer's software to manually count all keratocyte nuclei that were in focus; cells whose borders were not completely within the image frame were included on the top and right-hand aspects of the frame and excluded on the bottom and left-hand aspects. Keratocyte density was calculated as the number of cells per area scanned (i.e., $160 \mathrm{~mm}^{2}$ ); the two graders' density measurements were averaged for each of the six scans per eye, and the median of the three anterior stromal scans and the median of the three posterior stromal scans were used for analysis.

\section{Procedure}

After topical anesthesia using proparacaine 0.5\% eye drops, a baseline measurement of the corneal thickness was performed using the Pacscan 300P ultrasonic pachymetry device (Sonomed, New York, USA ). This measurement was then repeated after debriding the central $8 \mathrm{~mm}$ of the corneal epithelium. A metal guard was placed at the limbal region to protect the stem cells from the UV radiation and also to act as a well for the instilled riboflavin solution. Distilled water was instilled every $2 \mathrm{~min}$ for $10 \mathrm{~min}$ in order to swell the cornea, which was confirmed by pachymetry. This was followed by instillation of $0.1 \%$ hypotonic riboflavin solution every 2 min for 20 min. Corneal thickness measurements were repeated after application of 
riboflavin at the thinnest point of the cornea to confirm a value $>400 \mu \mathrm{m}$. In cases where the thickness was $<400 \mu \mathrm{m}$, hypotonic riboflavin was instilled every $20 \mathrm{sec}$ for another $5 \mathrm{~min}$ until the thickness increased to $\geq 400 \mu \mathrm{m}$. All cases in this report eventually reached a central thickness of $\geq 400 \mu \mathrm{m}$. Subsequently, ultraviolet light of 370 $\mathrm{nm}$ wavelength was used to irradiate an $8 \mathrm{~mm}$ diameter of the central cornea with an irradiance of $3 \mathrm{~mW} / \mathrm{cm}^{2}$ (using the UV-X device, UV-X; Company Peschke, Nürnberg, Germany). During the 30 min of irradiation, hypotonic riboflavin solution was instilled to the cornea every 2 min to maintain the necessary concentration of the solution and the required thickness of the cornea. A drop of $0.5 \%$ moxifloxacin solution was instilled at the end of the procedure, followed by application of a bandage soft contact lens (BCL) and instructions to apply moxifloxacin $0.5 \%$ four times per day. On the third postoperative day, the BCL was removed and topical loteprednol $0.5 \%$ four times per day was added on the seventh postoperative day, which was tapered over three weeks.

\section{Statistical Analysis}

The Wilcoxon signed rank test was used to test the difference between baseline and follow-up visits, with a significance level of 0.05 for each analysis. No correction was made for multiple comparisons in this small hypothesis-generating study. All statistical analyses were performed using Stata 14.2 (College Station, TX, USA).

\section{RESULTS}

The study population included four females and six males, with a median age of 18 years (interquartile range [IQR] 14-20; range, 12-21). Four patients had a previous episode of VKC. The median thinnest corneal pachymetry measurement on Pentacam was 381 Jm (IQR 367-390; range, 350-399).

\section{Corneal Topography}

The median Kmax on Pentacam was 61.4 diopters (IQR 55.2-67.4) preoperatively and 57.2 diopters (IQR 52.5-66.1) at the six-month postoperative visit; $P=0.11$. The keratometry estimate decreased by a median of 1.1 diopters (IQR 3.2-diopter decrease to 0.2-diopter increase) over the six-month period, with five subjects exhibiting regression (defined as a reduction of Kmax of $\geq 1$ diopter), one subject developing progression (defined as an increase of $\mathrm{Kmax}$ of $\geq 1$ diopter), and the remainder maintaining a relatively stable topography (change in Kmax of $<1$ diopter).

\section{In Vivo Confocal Microscopy (IVCM)}

The findings noted in the central $8 \mathrm{~mm}$ of the keratoconic corneas are as follows:

Epithelium: All layers of the corneal epithelium showed normal cell morphology preoperatively in all corneas. Of note, the VKC patients did not show any additional distinct changes in the epithelium before treatment. At the one-month postoperative period, the epithelium was disorganized in all study subjects, with variably irregular cell shape and size compared to the preoperative images. At three months, these changes in epithelial cell architecture had started to resolve, with complete normalization in all corneas by six months [Figure 1]. Three corneas, two of which were from patients with VKC, displayed hyperreflective globular cells, that is, microdot cells between epithelial cells, preoperatively; all three corneas showed an increase in hyperreflectivity and size post CXL. In two additional patients with VKC, microdot cells were absent preoperatively but developed in the early post $\mathrm{CXL}$ period. In all cases, these hyper-reflective microdot cells decreased in number and size by six months.

\section{Sub-basal Nerve Plexus}

Preoperatively, 10 corneas had a tortuous and branching sub-basal nerve plexus, and 2 showed a beaded appearance as well. At the first postoperative month, the nerve plexus could not be visualized in any of the corneas. By three months, six (60\%) subjects exhibited at least some regeneration of the nerves, visualized as small, fragmented nerve fibers with neuritic flocculation. By six months, 8 of the 10 corneas had nearly total regeneration of the subepithelial nerve plexus, except that nerve interconnections were not complete [Figure 2]. In the other two corneas, nerve regeneration was slow and only small, fragmented structures were seen even at six months. Three corneas (30\%) showed 

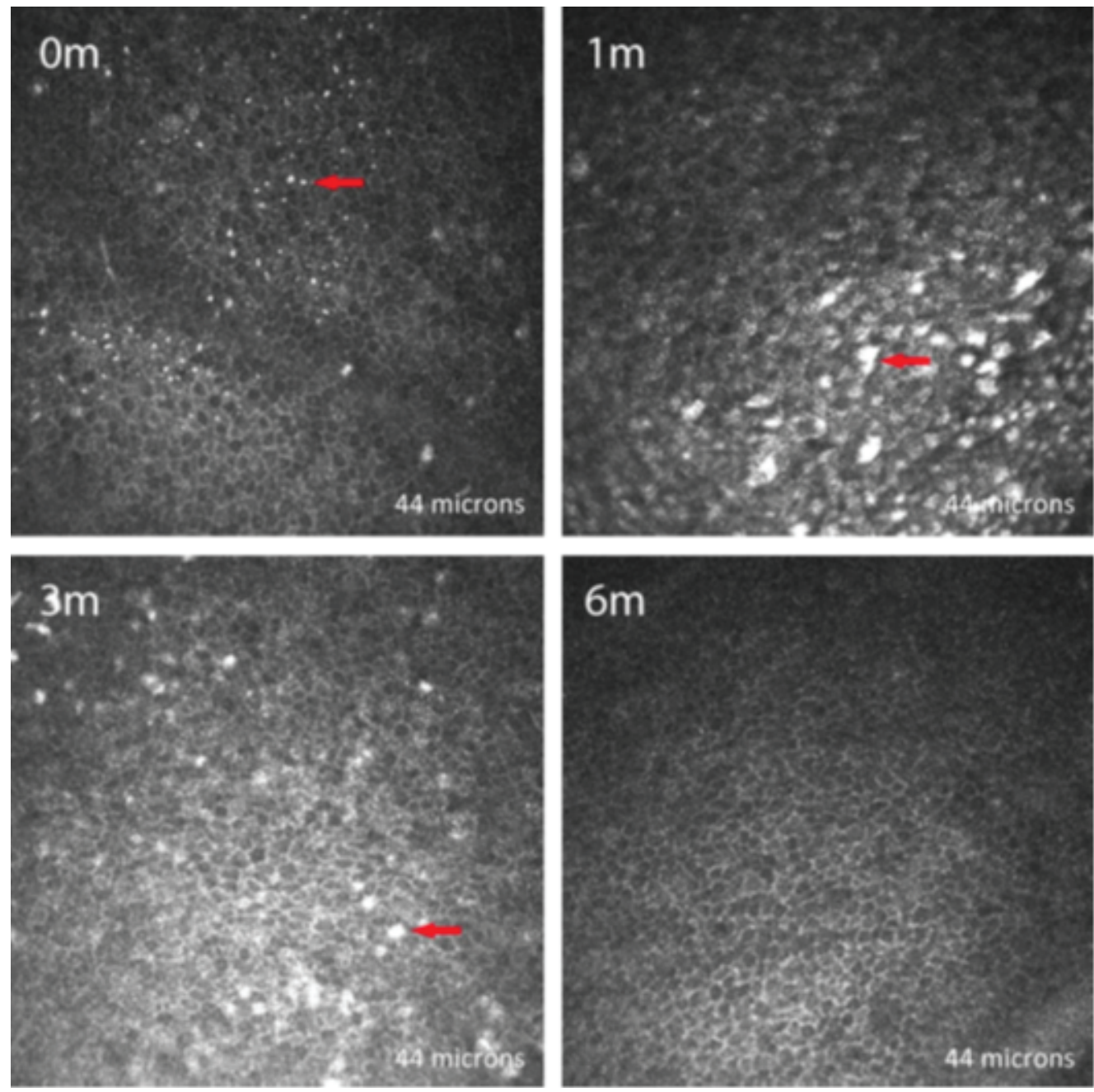

Figure 1. In vivo confocal microscopic images of the epithelium in a representative patient, taken at the same level at each visit. The epithelium appears normal preoperatively $(0 \mathrm{~m})$ which showed minimal disorganization at one month $(1 \mathrm{~m})$ and three months $(3 \mathrm{~m})$ but normalization at six months $(6 \mathrm{~m})$. Microdot cells (arrows) were hyperreflective and showed an increase in size at one month and gradually reverted to original state over time.

the presence of dendritic cells preoperatively, two of whom had VKC. In all three corneas, the dendritic cells appeared hyper-reflective and elongated one month after $C X L$, with a reduction in hyper-reflectivity at three months and absence of hyper-reflectivity at six months, at which time they appeared similar to their preoperative structure.

\section{Stroma}

At one month postoperatively, all corneas showed significant keratocyte apoptosis, with a honeycomb pattern that was more evident in the anterior to mid stroma. The posterior stroma of all corneas displayed dense hyper-reflective needle-shaped bands in a net-like pattern at the one-month visit. Keratocyte apoptosis extended up to the mid stroma in all corneas, and in four patients extended up to the posterior stroma, although the extensive hyper-reflectivity of the stroma made identification of keratocytes difficult. By three months, all subjects showed keratocytes repopulating the stroma, which was more evident in the anterior stroma. The hyper-reflective appearance of the anterior stroma was still evident but the honeycomb appearance had generally diminished. Hyper-reflective bands similar to those seen in the posterior stroma at one month were now seen at the mid stromal level. By six months post CXL, all subjects had a marked reduction in the hyper-reflectivity and complete absence of the honeycomb pattern in the anterior stroma. 

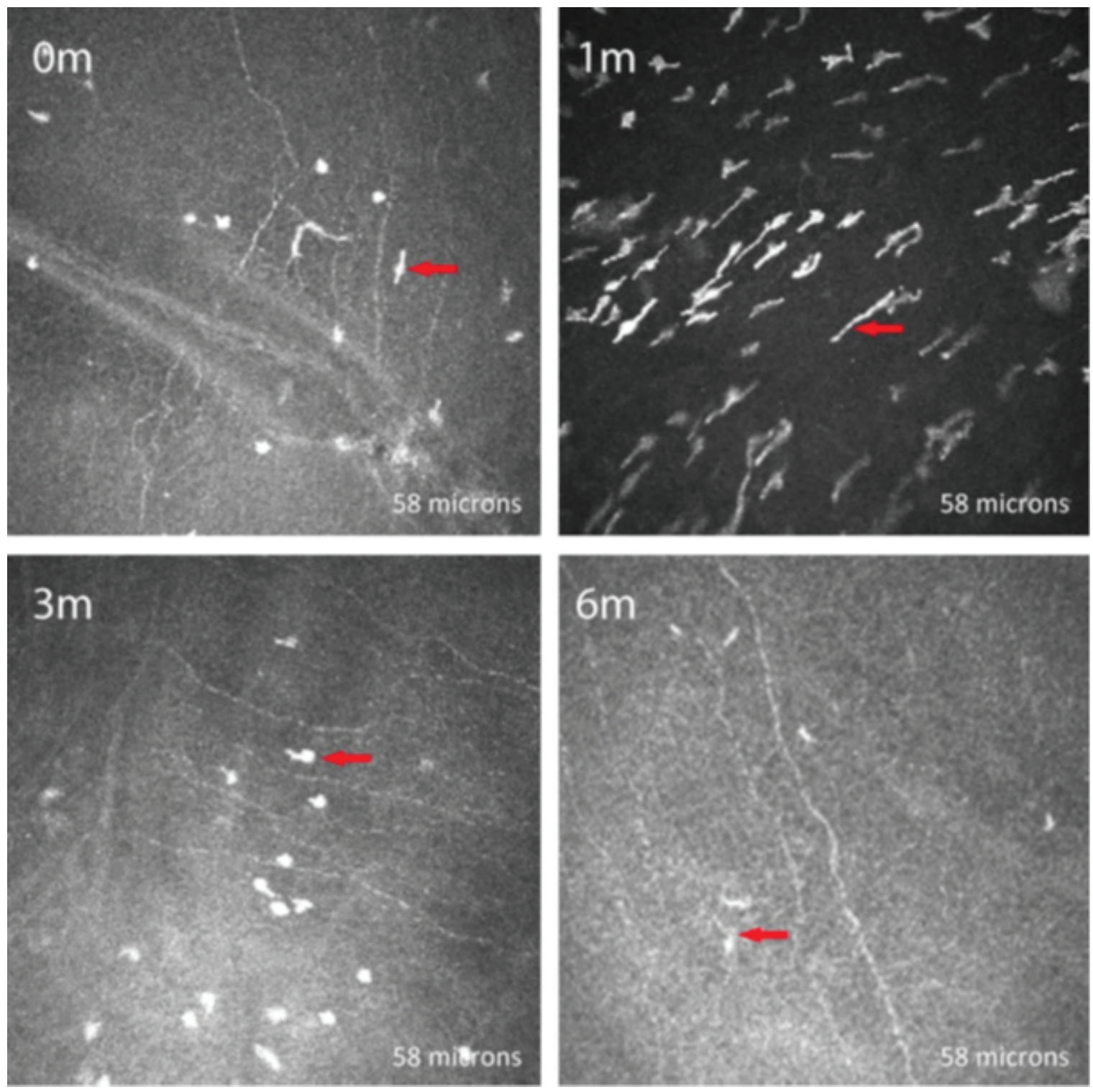

Figure 2. In vivo confocal microscopic images of Bowmans membrane in a representative patient, taken at the same level at each visit. The preoperative scan shows tortuous nerve fibers $(0 \mathrm{~m})$. Nerves appear fragmented at one month $(1 \mathrm{~m})$ and three months ( $3 \mathrm{~m}$ ); nerve regeneration is seen at six months $(6 \mathrm{~m})$. dendritic cells (arrows) are also seen, which appear hyper-reflective and elongated after crosslinking and revert to the original morphology by six months.

The stromal keratocyte population had generally increased compared to the previous visits [Figures 3 and 4], though four corneas continued to show deep posterior stromal keratocyte depletion [Figure 5].

\section{Keratocyte Density}

The median anterior stromal keratocyte density was 572 (IQR 497-681) preoperatively, decreased to 266 (IQR 110-385) at the threemonth postoperative visit $(P=0.005)$, and then increased to 368 (IQR 243-487) by six months $(P=0.04$ compared to three months and $P=$ 0.007 compared to preoperative) [Table 1]. The median preoperative posterior stromal keratocyte density was 264 (IQR 254-284), which decreased slightly, but not statistically significantly, at the three-month (median 202, IQR 71-281; $P=0.17$ ) and six-month visit (median 175, IQR 72-291; $P=0.96$ compared to the three-month visit and $P=0.14$ compared to preoperative) [Table 2]. 

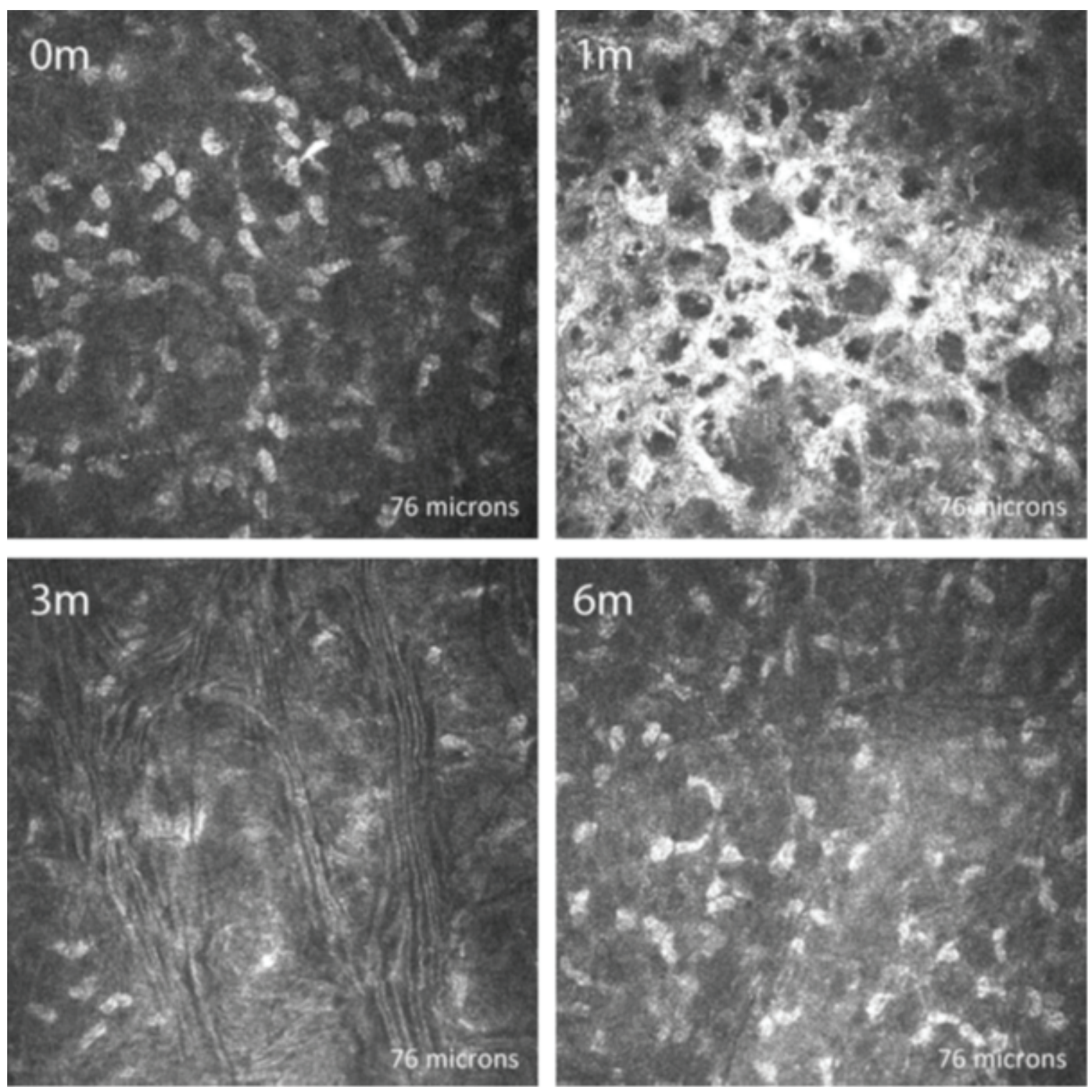

Figure 3. In vivo confocal microscopic images of the anterior stroma in a representative patient, taken at the same level at each visit. The scan shows normal keratocytes preoperatively $(0 \mathrm{~m})$. Post crosslinking, the anterior stroma showed hyper-reflective cytoplasm and extracellular lacunae in a honeycomb-like appearance with absence of keratocytes at one month $(1 \mathrm{~m})$ and gradual keratocyte regeneration at three months $(3 \mathrm{~m})$ and six months $(6 \mathrm{~m})$.

\begin{tabular}{|c|c|c|c|}
\hline \multicolumn{4}{|c|}{ Anterior stromal keratocyte density } \\
\hline & Median & IQR & P-value \\
\hline Pre-op & 572 & $497-681$ & - \\
\hline Three month & 266 & $110-385$ & 0.005 \\
\hline Six month & 368 & $243-487$ & 0.007 \\
\hline
\end{tabular}

$I Q R$, interquartile range 

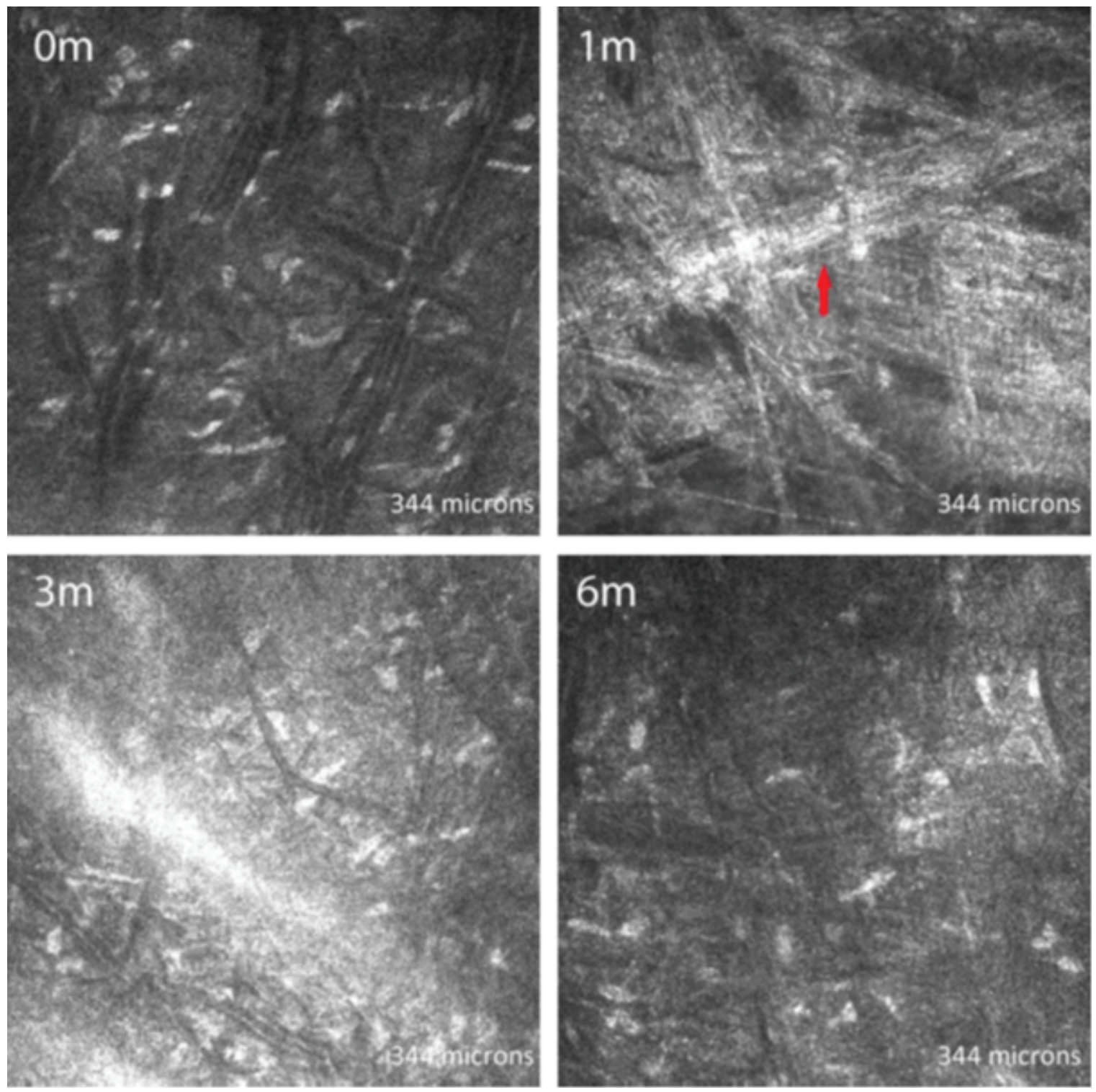

Figure 4. In vivo confocal microscopic images of the posterior stroma in a representative patient without posterior stromal apoptosis. Compared with the preoperative scan $(0 \mathrm{~m})$, the one-month scan shows new collagen fibers $(1 \mathrm{~m}$; arrow), but the keratocyte density is not affected at three months $(3 \mathrm{~m})$ neither at six months $(6 \mathrm{~m})$ after crosslinking.

Table 2. Quantitative analysis of posterior stromal keratocyte density before and after Hypotonic collagen crosslinking

\begin{tabular}{|c|c|c|c|}
\hline \multicolumn{4}{|c|}{ Posterior stromal keratocyte density } \\
\hline & Median & $I Q R$ & $P$-value \\
\hline Pre-op & 264 & $254-284$ & - \\
\hline Three month & 202 & $71-281$ & 0.17 \\
\hline Six month & 175 & $72-291$ & 0.14 \\
\hline
\end{tabular}

IQR, interquartile range 

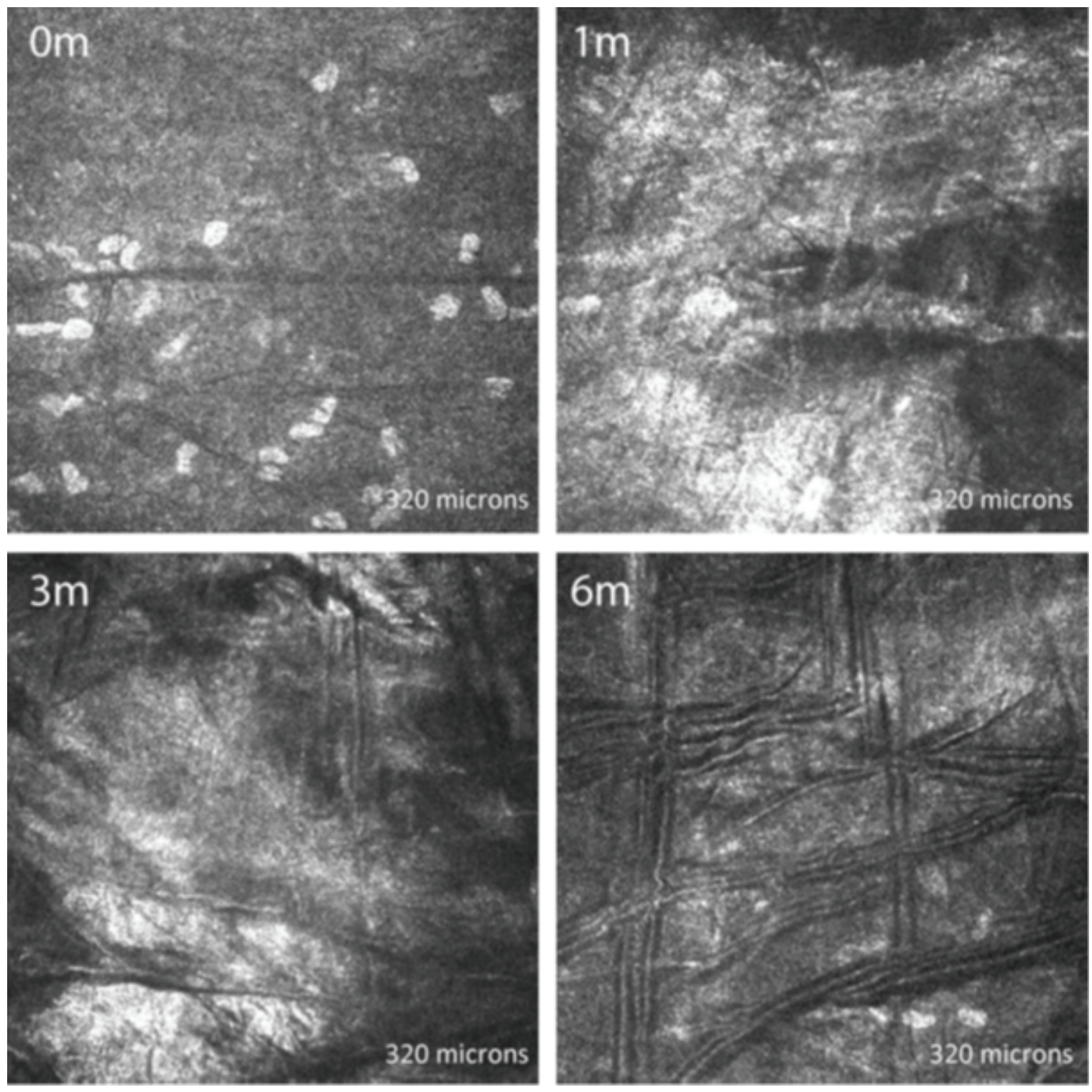

Figure 5. In vivo confocal microscopic images of the posterior stroma in a representative patient with posterior stromal apoptosis. Compared with the preoperative scan $(0 \mathrm{~m})$, a reduction in the number of keratocytes was observed at one month $(1 \mathrm{~m})$, three months (3 $\mathrm{m})$, and six months $(6 \mathrm{~m})$.

Table 3. Analysis of endothelial cell density before and after hypotonic collagen crosslinking

\begin{tabular}{lcccc}
\hline & Median $E C D\left(\mathrm{ce} / \mathrm{ls} / \mathrm{mm}^{2}\right)$ & IQR & P-value \\
\hline Pre-op & 2895 & $2786-2907$ & - \\
Post-op & 2660 & $2451-2793$ & 0.005 \\
\hline
\end{tabular}

ECD, endothelial cell density; IQR, interquartile range

\section{Endothelium}

The endothelial cell morphology was normal in all patients preoperatively and at all subsequent study visits [Figure 6].

\section{Confocal Findings of Progression}

One subject had progression of keratoconus over the six-month follow-up period, with an increase in Kmax of 6 diopters. This subject was 12 

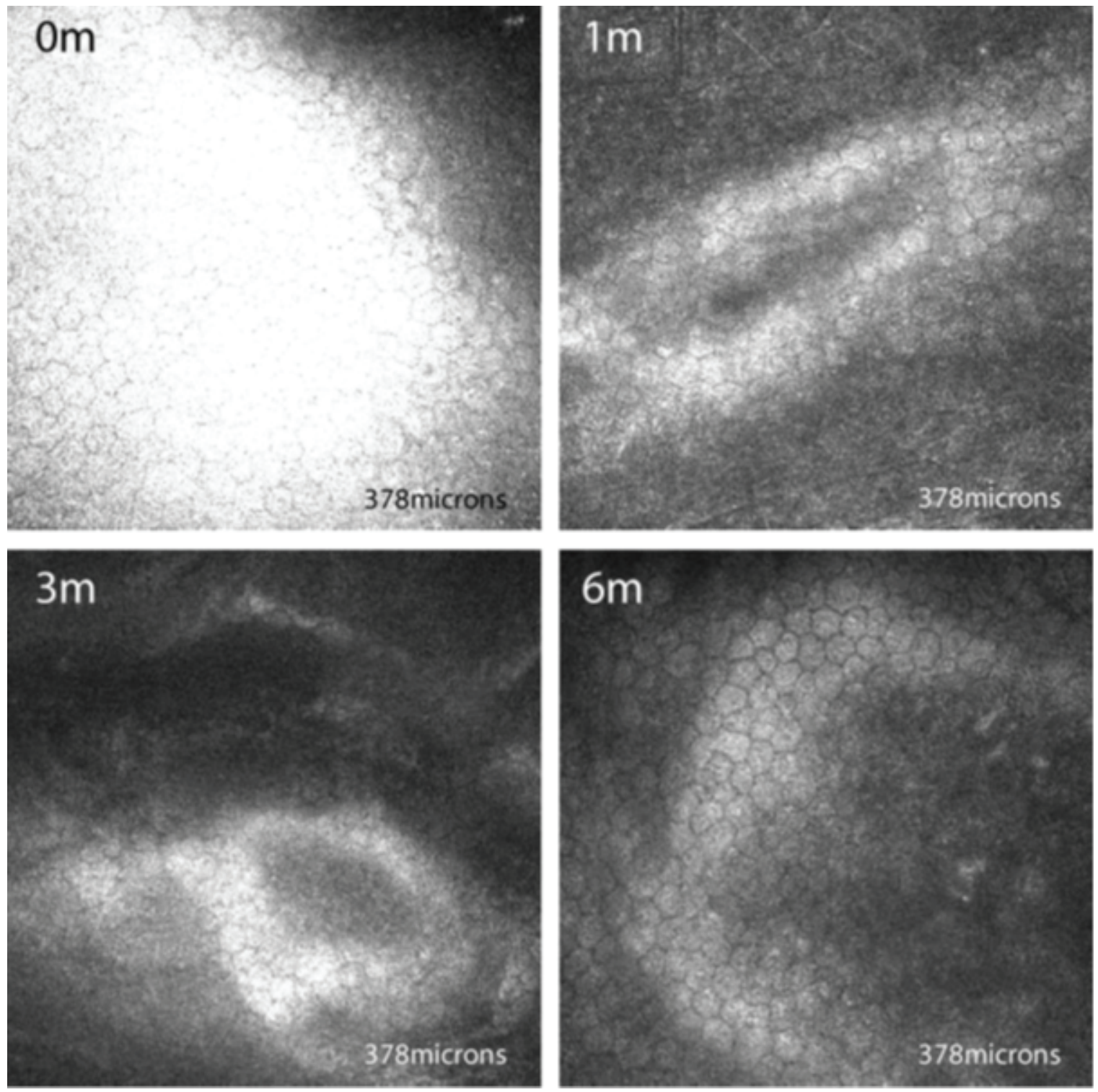

Figure 6. In vivo confocal microscopic images of the endothelium of a representative patient, taken at the same level at each visit. The endothelial morphology and density appeared normal preoperatively $(0 \mathrm{~m})$ and at one-, three-, and six-month visits $(0$ $\mathrm{m}, 3 \mathrm{~m}$, and $6 \mathrm{~m}$, respectively).

years old, had VKC, and had a family history of keratoconus. The confocal findings of this participant's cornea was most notable for having a very thin Bowman's membrane, breaks in the Bowman's membrane and also being one of the two described above cases that had poor nerve regeneration. Otherwise, confocal findings were similar to the corneas that did not experience progression.

Peripheral cornea: An area of inferior peripheral cornea outside the central treatment area was examined as an internal control. The epithelium and stroma of the peripheral cornea was normal in all study participants, with no evidence of treatment effects at any stages of the followup.

\section{Specular Microscopy}

Although all subjects had a reduction in endothelial cell density (median reduction 263 cells $/ \mathrm{mm}^{2}$, IQR 90-342), none showed any signs of endothelial damage clinically (i.e., corneal oedema). The median endothelial cell density as assessed by automated specular microscopy is depicted in Table 3. 


\section{DISCUSSION}

IVCM showed profound morphological changes in corneal cells and structures following hypotonic riboflavin CXL. After the procedure, epithelial cell morphology returned to normal by six months; however, the changes in the subepithelial nerve plexus, stroma, and keratocytes did not. Anterior keratocyte density was markedly reduced after $C X L$ and subsequently increased but did not return to normal by the six-month postoperative visit. Endothelial cell density decreased after CXL, although this reduction was not accompanied by morphological changes and did not seem to be clinically meaningful by six months of follow-up.

Although several studies have reported the IVCM findings after CXL on corneas $>400$ $\mu \mathrm{m}$, very few have documented the changes specifically for thin corneas (i.e., $<400 \mu \mathrm{\mu m}$ ). Those that have assessed thin corneas have studied techniques using isotonic riboflavin. ${ }^{[19-21]}$ Our study is novel because it documents the corneal changes post CXL in thin corneas treated with hypotonic riboflavin. While Raiskup ${ }^{[22]}$ and Fahedi ${ }^{[12]}$ have described the efficacy of $\mathrm{CXL}$ with hypotonic riboflavin in thin corneas, the corneal structural changes analyzed by IVCM have not yet been reported. latrogenic swelling of the cornea with hypotonic riboflavin solution increases the stromal thickness by $25 \%$ after 30 min. ${ }^{[23]}$ Hypotonic riboflavin CXL takes advantage of this phenomenon in an attempt to prevent the crosslinking procedure from damaging the endothelium.

The epithelial changes in hypotonic CXL were generally similar to that of isotonic $\mathrm{CXL}$, with transient changes that returned to normal within six months. However, patients with VKC showed distinct structural features including presence of microdot cells, and dendritic cells, which became more pronounced after CXL and gradually reverted to their quiescent state over three to six months post CXL. Previous studies have described these cells in normal individuals and those with VKC, ${ }^{[24]}$ and other inflammatory conditions like post Femtosecond Laser-assisted Keratoplasty ${ }^{[25]}$ and contact lens use, ${ }^{[26]}$ but we are not aware of any reports of their appearance post CXL. Their presence did not seem to correlate with topographic changes post CXL, however, since they are thought to indicate inflammation, they may be worthy of further study in patients with VKC and $\mathrm{KCN}$.

Previous studies of isotonic CXL have reported loss of sub-basal nerves in the early postoperative period, with regeneration as disconnected nerves between the second and third postoperative months, and eventually the appearance of interconnected fibers at 6-12 months. ${ }^{[15,17,18]}$ In contrast to what has been reported for isotonic $\mathrm{CXL}$, sub-basal nerve regeneration after hypotonic CXL was not uniform in our patients. Two of the ten subjects showed very slow nerve regeneration, with a short, fragmented appearance and low density even at six months. It is unclear whether this response represents an expected variation in the healing response, or if this reflects a difference in response to hypotonic riboflavin.

A marked stromal honeycomb pattern suggestive of edema was present one month postoperatively and gradually decreased over the next months until completely absent by six months. This honeycomb pattern was seen more in the mid stroma than in the anterior stroma, possibly because the anterior stroma is more compact compared to the mid stroma. The posterior stroma contained hyper-reflective bands at the one-month post-CXL visit, suggestive of new collagen fibers synthesized by the activated keratocytes. By three months, similar-appearing bands were seen at the anterior mid stromal level. This confocal observation has been suggested to be due to anterior migration of the collagen bands. ${ }^{[27]}$

Some studies of isotonic riboflavin CXL have used changes in keratocyte density to document a demarcation line around 300-320 $\mu \mathrm{m}$ posterior to the epithelium that marks the transition from the treated zone to the untreated zone. Studies of thin corneas have recorded this demarcation line at a slightly deeper location, approximately 320-350 $\mu \mathrm{m}$ posterior to the epithelium. ${ }^{[19,20]}$ It was difficult for us to comment on such a demarcation line since the hyperreflectivity of the stroma precluded an easily visible transition zone.

Although the IVCM analysis of the endothelium did not show any morphological changes at any point postoperatively, the endothelial cell count density was significantly lower at six months than at the preoperative visit. The impact of this finding is not clear. Given the treatment of such thin corneas, it is possible that the crosslinking procedure caused endothelial damage that was not evident 
morphologically on confocal microscopy. Similar reductions have been observed in some studies of isotonic riboflavin $\mathrm{CXL}^{[28]}$ and also in one study of thin corneas ${ }^{[19]}$ but not in any others. ${ }^{[19,20]}$ None of our patients developed corneal edema at any stages of the follow-up, suggesting that the reduction in endothelial cell density was not clinically meaningful over the six months of the study. Nevertheless, this is an important finding that should be studied further.

Many of the findings in the present study were similar to those reported previously following standard or isotonic CXL treatment, including the development and resolution of stromal hyper-reflectivity and stromal honeycomb morphology, as well as the sub-epithelial nerve plexus destruction and regeneration. However, we also noted some differences. The most important difference was the depth of keratocyte apoptosis. Previous studies of isotonic riboflavin $C X L,{ }^{[13-15]}$ as well as those specifically performed on thin corneas with $\mathrm{CACX} \mathrm{L}^{[19]}$ and customized epithelial debridement ${ }^{[20,21]} \mathrm{CXL}$ have described structural changes and keratocyte apoptosis limited to anterior to mid stroma. In contrast, we found keratocyte apoptosis extending to mid stroma in six subjects, and to posterior stroma in four subjects assessed by the presence of apoptotic cells in posterior stroma and also by the decreased keratocyte density in posterior stroma in these patients post CXL. We speculate that this could be attributed to fluctuating corneal hydration during the CXL procedure with hypotonic CXL. In addition, we noticed marked dendritic and microdot globular cell activation not previously observed in isotonic riboflavin CXL studies; however, these changes mostly occurred in patients with VKC. It is difficult to determine whether the differences are due to differences in patient populations or surgical technique.

The chief limitation of our study is the small number of subjects included. The efficacy and safety of this technique in the long-term needs to be assessed in subsequent studies with a greater number of patients. Also, four patients in our study had history of vernal disease, which could have confounded our findings. Generalizations are difficult to make given the heterogeneous nature of this cohort.

In conclusion, thin corneas treated with hypotonic CXL generally had same confocal microscopy findings as previous reports of isotonic $C X L$, with the exception of more posterior keratocyte apoptosis in the hypotonic procedure. This more posterior involvement was accompanied by a significant reduction in endothelial cell density, although not by changes in endothelial cell morphology neither by corneal oedema clinically. Further study of hypotonic CXL is warranted to assess the risk of damage more accurately to the endothelium in patients with corneas $<400 \mu \mathrm{m}$ undergoing $\mathrm{CXL}$.

\section{Financial Support and Sponsorship}

None.

\section{Conflicts of Interest}

The authors have no conflicts of interest to declare.

\section{REFERENCES}

1. Rabinowitz Y. Keratoconus. Surv Ophthalmol 1998;42:297-319.

2. Tuori AJ, Virtanen I, Aine E, Kalluri R, Miner JH, Uusitalo $\mathrm{HM}$. The immunohistochemical composition of corneal basement membrane in keratoconus. Curr Eye Res 1997;16:792-801.

3. Cheng EL, Maruyama I, Sundar Raj N, Sugar J, Feder RS, Yue BY. Expression of type XII collagen and hemidesmosome-associated proteins in keratoconus corneas. Curr Eye Res 2001;22:333-340.

4. Cannon DJ, Foster CS. Collagen crosslinking in keratoconus. Invest Ophthalmol Vis Sci 1978;17:6365.

5. Spoerl E, Huhle M, Seiler T. Induction of cross-links in corneal tissue. Exp Eye Res 1998;66:97-103.

6. Wollensak G, Spoerl E, Seiler T. Riboflavin/ultravioletA induced collagen crosslinking for the treatment of keratoconus. Am J Ophthalmol 2003;135:620-627.

7. Wittig-Silva $C$, Whiting $M$, Lamoureux $E$, Lindsay RG, Sullivan LJ, Snibson GR. A randomized controlled trial of corneal collagen cross-linking in progressive keratoconus: preliminary results. J Refract Surg 2008;24:720-725.

8. Spoerl E, Mrochen M, Sliney D, Trokel S, Seiler T. Safety of UVA riboflavin cross-linking of the cornea. Cornea 2007;26:385-389.

9. Caporossi A, Mazzotta C, Baiocchi S, Caporossi T. Longterm results of riboflavin ultraviolet $A$ corneal collagen cross-linking for keratoconus in Italy: The Siena Eye Cross Study. Am J Ophthalmol 2010;149:585-593.

10. Raiskup WF, Hoyer A, Spoerl E, Pillunat LE. Collagen crosslinking with riboflavin and ultraviolet-A light in keratoconus: long-term results. J Cataract Refract Surg 2008;34:796801. 
11. Raiskup F, Spoerl E. Corneal crosslinking with riboflavin and ultraviolet A. Part II. Clinical indications and results. Ocul Surf 2013;11:93-108.

12. Hafezi F, Mrochen M, Iseli HP, Seiler T. Collagen crosslinking with ultraviolet-A and hypoosmolar riboflavin solution in thin corneas. J Cataract Refract Surg 2009;35:621-624.

13. Cavanagh HD, Petroll WM, Alizadeh H, He YG, McCulley JP, Jester JV. Clinical and diagnostic use of in vivo confocal microscopy in patients with corneal disease. Ophthalmology 1993;100:1444-1454.

14. Petroll WM, Cavanagh HD, Jester JV. Three-dimensional imaging of corneal cells using in vivo confocal microscopy. J Microsc 1993;170:213-219.

15. Kymionis GD, Diakonis VF, Kalyvianaki M, Portaliou D, Siganos C, Kozobolis VP, et al. One-year follow-up of corneal confocal microscopy after corneal cross-linking in patients with post laser in situ keratomileusis ectasia and keratoconus. Am J Ophthalmol 2009;147:774-778.

16. Mazzotta C, Caporossi T, Denaro R, Bovone C, Sparano C, Paradiso A, et al. Morphological and functional correlations in riboflavin UV A corneal collagen crosslinking for keratoconus. Acta Ophthalmol 2012;90: 259265.

17. Jordan C, Patel DV, Abeysekera N, McGhee CN. In vivo confocal microscopy analyses of corneal microstructural changes in a prospective study of collagen cross-linking in keratoconus. Ophthalmology 2014;121:469-474.

18. Mazzotta C, Traversi C, Baiocchi S, Caporossi O, Bovone $\mathrm{C}$, Sparano MC, et al. Corneal healing after riboflavin ultraviolet-A collagen cross-linking determined by confocal laser scanning microscopy in vivo: early and late modifications. Am J Ophthalmol 2008;146:527-533.

19. Mazzotta C, Jacob S, Agarwal A, Kumar DA. In vivo confocal microscopy after contact lens-assisted corneal collagen cross-linking for thin keratoconic corneas. $J$ Refract Surg 2016;32:326-331.

20. Kaya V, Utine CA, Yilmaz OF. Efficacy of corneal collagen cross-linking using a custom epithelial debridement technique in thin corneas: a confocal microscopy study. $J$ Refract Surg 2011;27:444-450.

21. Mazzotta C, Ramovecch V. Customized epithelial debridement for thin ectatic corneas undergoing corneal cross-linking: epithelial island cross-linking technique. Clin Ophthalmol 2014;8:1337-1343.

22. Raiskup F, Spoerl E. Corneal cross-linking with hypoosmolar riboflavin solution in thin keratoconic corneas. Am J Ophthalmol 2011;152:28-32.

23. Wollensak G, Aurich H, Wirbelauer C, Sel S. Significance of the riboflavin film in corneal collagen crosslinking. $J$ Cataract Refract Surg 2010;36:114-120.

24. Mastropasqua L, Nubile $M$, Lanzini $M$, Carpineto $P$, Ciancaglini M, Pannellini T, et al. Epithelial dendritic cell distribution in normal and inflamed human cornea: in vivo confocal microscopy study. Am J Ophthalmol 2006;142:736-744.

25. Shtein RM, Kelley KH, Musch DC, Sugar A, Mian SI. In vivo confocal microscopic evaluation of corneal wound healing after femtosecond laser-assisted keratoplasty. Ophthalmic Surg Lasers Imaging 2012;43:205-213.

26. Böhnke M, Masters BR. Long-term contact lens wear induces a corneal degeneration with microdot deposits in the corneal stroma. Ophthalmology 1997;104:1887-1896.

27. Kymionis GD, Portaliou DM, Diakonis VF, Kontadakis GA, Krasia MS, Papadiamantis AG, et al. Posterior linear stromal haze formation after simultaneous photorefractive keratectomy followed by corneal collagen crosslinking. Invest Ophthalmol Vis Sci 2010;51:5030-5033.

28. Razmjoo H, Ghoreishi SM, Mohammadi Z, Salam $H$, Nasrollahi K , Peyman A. Comparison of the findings of endothelial specular microscopy before and after corneal cross-linking. Adv Biomed Res 2015;4:52.

29. Cagil N, Sarac O, Can GD, Akcay E, Can ME. Outcomes of corneal collagen crosslinking using a customized epithelial debridement technique in keratoconic eyes with thin corneas. Int Ophthalmol 2017;37:103-109. 